\title{
Neurodevelopmental Effects of Undernutrition and Placental Underperfusion in Fetal Growth Restriction Rabbit Models
}

Míriam Illa ${ }^{1 *}$, Elisenda Eixarch ${ }^{1,2}$, Emma Muñoz-Moreno ${ }^{1,4}$, Dafnis Batalle ${ }^{1,5}$, Rocío Leal-Campanario ${ }^{3}$, Agnès Gruart $^{3}$, José María Delgado-García ${ }^{3}$, Francesc Figueras ${ }^{1,2}$, Eduard Gratacós ${ }^{1,2}$

${ }^{1}$ Fetal i+D Fetal Medicine Research Center, BCNatal - Barcelona Center for Maternal-Fetal and Neonatal Medicine (Hospital Clínic and Hospital Sant Joan de Déu), IDIBAPS, University of Barcelona, Spain.

${ }^{2}$ Centre for Biomedical Research on Rare Diseases (CIBER-ER), Spain.

${ }^{3}$ Division of Neurosciences, Pablo de Olavide University, Seville-41013, Spain.

4 Experimental MRI 7T Unit, Institut d'Investigacions August $\mathrm{Pi}$ iSunyer (IDIBAPS), Barcelona, Spain.

${ }^{5}$ Centre for the Developing Brain, Division of Imaging Sciences \& Biomedical Engineering, King's College London, London, SE17EH, United Kingdom.

*Short title: FGR effects in neurodevelopment

\section{${ }^{*}$ Corresponding Author:}

Miriam Illa Armengol, MD

Fetal i+D Fetal Medicine Research Center, BCNatal - Barcelona Center for Maternal-Fetal and Neonatal Medicine (Hospital Clínic and Hospital Sant Joan de Déu), IDIBAPS, University of Barcelona, Spain.

Sabino de Arana 1, 08028 Barcelona, Spain.

Tel. +34 932275400 EXT. 7339

Email: miriamil@clinic.ub.es 


\section{Abstract}

Introduction: Chronic reduction of oxygen and nutrient delivery to the fetus has been related to neurodevelopmental problems. Placental underperfusion induces a significant reduction in oxygen and nutrient delivery, whereas maternal undernutrition causes mainly nutrient deficiency. A comparison of the neurodevelopmental effects of both situations in pregnant rabbits was performed.

Material and Methods: The placental underperfusion model was induced after uteroplacental vessel ligation at 25 days of pregnancy. The undernutrition model was induced after a reduction of $70 \%$ of the basal maternal intake at 22 days of pregnancy. Neurobehavioral tests were applied in the derived offspring at the neonatal period and at long-term. Structural brain differences were evaluated by brain networks obtained from diffusion magnetic resonance imaging.

Results: Birth weight was significantly lower in both cases. However, stillbirth was only increased in the placental underperfusion model. Cases from both models presented poorer neurobehavioral performance and network infrastructure, being more pronounced in the placental underperfusion model.

Discussion: Prenatal insults during the last third of gestation resulted in functional and structural disturbances. The degree of neurodevelopmental impairment and its association with structural brain reorganization seemed to be related to the type of the prenatal insult, showing stronger effects in the placental underperfusion model.

Key words: Animal model, behavior, brain networks, fetal growth restriction, placental insufficiency, undernutrition.

\section{Abbreviations:}

FA: fractional anisotropy; FGR: fetal growth restriction; MRI: magnetic resonance imaging; OFBT: open field behavioral test; ORT: object recognition task; PU: placental underperfusion; UN: undernutrition. 


\section{Introduction}

Fetal growth restriction (FGR) has been related to neurobehavioral problems during the childhood period [1, 2] that also persist long term [3, 4]. Interestingly, long-term follow-up studies have described cognitive impairments and learning difficulties at school age [5] involving short-term memory, and attention and anxiety problems [3, 4].

Although this evidence exists, the exact mechanisms underlying fetal brain programming due to FGR are still unknown. Notwithstanding their obvious limitations, animal models provide an opportunity to advance the investigation of the pathophysiology of this condition. There are two major approaches to reproduce preclinical FGR models: models based in placental underperfusion (PU) and models based in undernutrition (UN) [6]. PU models are based on the reduction of uteroplacental blood flow, which decreases supply of both oxygen and nutrients, whereas UN models are based on the reduction of maternal intake, which results mainly in a decrease of nutrients. Regarding PU models, different methods have been described. While generalized reduction in the uteroplacental blood supply by means of uteroplacental embolization and bilateral uterine artery ligature resulted in nonpredictable reductions of placental perfusion [7], selective ligature of uteroplacental vessels performed in pregnant rabbits has been demonstrated to develop a gradable model of FGR [8]. Regarding UN models, maternal food deprivation has also demonstrated human FGR characteristics in pregnant rabbits $[9,10]$.

Although nutrients and oxygen have been described to be essential elements needed to complete normal fetal brain programming [11], we hypothesized that neurodevelopmental effects of oxygen and nutrient restriction during fetal neurodevelopment would have a greater impact on brain programming in comparison to a nutrient deficiency alone. For that purpose, the current study attempts to describe the neurodevelopmental impact of what are probably the two major causes worldwide of adverse intrauterine environment affecting the biologic growth potential (PU model by means of uteroplacental vessel ligation and UN model during pregnancy) induced during the last third of gestation [8]. In the offspring derived from these models, neurobehavioral tests at the neonatal and long-term period and structural brain changes using 
structural brain networks from ex-vivo diffusion magnetic resonance imaging (MRI) at the long-term period were evaluated. 


\section{Materials and Methods}

The methodology of the study and flow chart are summarized in Figure 1.

\section{Animals, FGR induction, and ethics statement}

FGR was induced in pregnant rabbits during the last third of gestation following two different approaches: placental underperfusion (PU) and undernutrition (UN). The PU model was induced in nine pregnant rabbits following the detailed protocol previously described [8]. Briefly, ligation of $40 \%$ to $50 \%$ of the uteroplacental vessels of all the gestational sacs of one horn was performed at 25 days of pregnancy, whereas the contralateral horn was used as a control. At 30 days of pregnancy, a cesarean section was performed. The UN model was performed in three pregnant rabbits, reducing $70 \%$ of the basal food intake, corresponding to approximately $45 \mathrm{~g} /$ day of the standard chow specifically designed for the pregnant and lactating rabbit mother (2030 Teklad Global Rabbit Diet). Basal food reduction was started from 22 days of gestation up to 31 days of gestation, when a vaginal delivery was allowed [12]. As a control for the UN model, we included three rabbits that were feed ad libitum. Vaginal delivery was allowed or induced with oxytocin at 31 days of pregnancy. Pups from both models were weighed and identified with a subcutaneous chip. The PU model pups were housed and breastfed by a surrogate, whereas in the UN model, pups were fed by their own mother, with a maximum of eight pups in both models. Pups were housed until the 30th postnatal day, when they were weaned. Thereafter, both groups of rabbits were housed in groups of three with a reversed 12/12h light cycle, and free access to water and food. The animal experimentation in this study was approved by the Animal Experimental Ethics Committee of the University of Barcelona (permit number: 206/10-5440).

\section{Functional tests protocol evaluation}

At postnatal day 1, neurobehavioral evaluation was performed following the methodology previously described [13,14], evaluating general motor skills, reflexes, and olfactory sensitivity.

Between postnatal days 60 and 70, evaluation of learning, anxiety, and memory was performed. Concerning the learning evaluation, a Skinner box was 
constructed as detailed in Leal-Campanario et al. (box for operant conditioning and instrumental learning for rabbits, 2012. Inscription number in Spain: P2001231369), and the protocol was adapted from the methodology previously described [15] with food reward reinforcement and a continuous reinforcement schedule. One week before starting the evaluation, rabbits were food-deprived ( $20 \mathrm{~g} / \mathrm{d}$ of food chow) to increase their motivation to get the food reward. After observing a $10 \%$ to $15 \%$ reduction in their basal weight, the first shaping phase was started. This phase lasted 5 days, and any advancement toward the feeder bar was rewarded. After 2 days of rest, the training phase lasting 5 more days was performed, and a reward was given only when the animal specifically pressed the lever. In this phase, the learning criterion was considered to be when the animal pressed the lever and went directly toward the food dispenser to obtain the reward at least three times in one session. All sessions lasted 10 minutes and were recorded for posterior analysis. After the Skinner test, the animals were allowed to rest for 2 days before continuing with the Open Field Behavioral Test (OFBT) and the Object Recognition Task (ORT), respectively. These tests were applied following methodology published previously [16], and examinations were recorded and analyzed using a video tracking software (SMART Software Tracking System from Panlab). OFBT variables included "latency" (time in seconds the animal leaves the familiar starting point and starts exploring the open field) and "number of internal and external areas explored." Variables recorded in the ORT included: time in seconds exploring the two objects presented in the familiarization phase and time in seconds exploring the familiar and the novel objects in the testing phase. The discriminatory index (DI) was then calculated as follows:

$$
D I=\frac{\text { time exploring novel object }- \text { time exploring the familiar one }}{\text { time exploring novel object }+ \text { time exploring the familiar one }}
$$

After the neurobehavioral tests, rabbits were anesthetized (Ketamina + Xylacine) and euthanized with an endovenous overdose of sodium pentobarbital. Brains were then fixed with $10 \%$ buffered formalin solution through a cardiac perfusion fixation protocol. After fixation, brains were removed and placed in $10 \%$ buffered formalin solution overnight. 


\section{MRI acquisition, tractography, brain parcellation, network extraction, and analysis}

A sub-sample of fixed brains was selected to be scanned (16 animals from PU and 14 from the UN). MRI was performed using a 7T animal MRI scanner (BrukerBioSpin MRI GmbH). Due to technical issues, high-resolution three-dimensional T1 weighted images were obtained in the UN's brain samples by a modified driven equilibrium Fourier transform (MDEFT) sequence; whereas T2-weighted images were obtained in the PU's brain samples by a rapid acquisition with relaxation enhancement (RARE) sequence. In both models, diffusion-weighted images (DWI) were acquired using a diffusion sequence covering 30 gradient directions with a b-value of $3000 \mathrm{~s} / \mathrm{mm}^{2}$ together with a baseline ( $\left.b=0 \mathrm{~s} / \mathrm{mm}^{2}\right)$ image. Pre-processing, tractography, brain parcellation, and brain network extraction were performed following the methodology previously described [17], obtaining an FA-weighted network for each subject. The Brain Connectivity Toolbox was used to characterize global functioning of each network by means of graph theory network features [18]. Particularly, we assessed infrastructure (average strength), integration (global efficiency), and segregation (local efficiency) of each FA-weighted network (see Supplementary material for a more detailed methodology explanation).

\section{Statistics}

Statistical comparisons were performed by general linear models (GLM) and were adjusted by gender in long-term data. Interaction of group (controls and cases) and gender was first included into the model, but as it did not show any significant effect, was excluded of the final model. Significance was declared at $\mathrm{p}<0.05$ (uncorrected). Normality was assessed by Shapiro-Wilk test and homoscedasticity by Levene's test, and when the null hypothesis was rejected, log-transformation was performed before GLM analysis. Descriptives of the variables were expressed as mean and standard deviation for normal distributions, whereas median and interquartile range were used for non-normal distributions. To evaluate functional impairments in both models, the mean difference (mean result in controls minus mean result in cases) and its $95 \% \mathrm{Cl}$ were calculated for each model and for each functional variable. Association of network features with functional results was performed by means of a partial 
correlation or GLM, as needed. The software package STATA13.0 was used for the statistical analyses. 


\section{Results}

\section{Perinatal data}

Stillbirth was statistically higher in cases coming from the PU model compared to their respective controls ( $44 \%$ vs. $5 \%, p<0.001)$. No stillbirth was observed in the UN model. Postnatal mortality rate did not differ between cases and controls in both models ( $42 \%$ vs. $32 \%, p=0.55$ in PU; $6 \%$ vs. $5 \%, p=0.94$ in UN, cases vs. controls, respectively). Regarding birth weight, both models had a similar effect, observing a significant birth weight decrease in cases compared to their respective controls (Figure 2). The degree of growth restriction induced by both models was similar [birth weight in PU: $30.23 \mathrm{~g}$ (SD 12.08); birth weight in UN: $51.92 \mathrm{~g}$ (SD 7.57)], as both FGR's birth weights corresponded to their $10^{\text {th }}$ percentile derived from normal birth weight distribution $\left(10^{\text {th }}\right.$ percentile from PU: $33 \mathrm{~g} ; 10^{\text {th }}$ percentile from UN: $53 \mathrm{~g}$ ).

At the long-term period evaluation, no differences in weight [1444 g (SD 136) vs. $1589 \mathrm{~g}$ (SD 376), $\mathrm{p}=0.24$ in PU; $1378 \mathrm{~g}$ (SD 101) vs. $1442 \mathrm{~g}$ (SD 111), $p=0.17$ in UN, cases vs. controls, respectively] and gender distribution (\% of females: $55 \%$ vs. $62 \%, p=0.73$ in PU; $33 \%$ vs. $31 \%$ in UN, cases vs. controls, respectively) were observed in these models.

\section{Functional results}

At the neonatal period, cases from both models showed poorer results in almost all the parameters (Supplementary Table S1). These differences were more pronounced in the PU model, as shown in Figure 3, where mean difference and its $95 \% \mathrm{Cl}$ for each functional variable were higher in the PU model.

At the long-term period, all the animals that reached that period did not present any motor abnormality that could have interfered in the execution of the neurobehavioral tasks. Due to technical problems, Skinner tests and OFBT were not available in 11 animals from PU and 8 animals from UN. ORT was attempted in all animals with a successful OFBT test; however, only 17 animals from the PU and 23 animals from the UN model were suitable to be included in the ORT analyses, since they explored at least one object in the Familiarization 
phase and at least one time both objects in the Testing phase [19]. Skinner test results showed a lower proportion of cases from the PU model reaching the learning criteria when compared with their controls (30\% vs. $77 \%, p=0.03$ cases vs. controls, respectively), whereas no differences were observed in the UN model ( $44 \%$ vs. $56 \%, p=0.56$ cases vs. controls, respectively). Regarding OFBT results, cases from both models presented a significantly increased latency of leaving the familiar starting point and a reduced number of external and internal boxes explored. When ORT was assessed, a decreased DI was observed in cases compared to their respective controls in both models (Supplementary Table S2). Again, these differences at the long-term period were more pronounced in the PU model, as shown in Figure 4, where mean difference and its $95 \% \mathrm{Cl}$ for each functional variable were higher in the PU model compared with the UN model.

\section{Brain network results}

Overall, animals with a history of FGR presented a significant decrease in brain network parameters when compared with their respective controls in both models at the long-term period. Regarding global and local efficiencies, cases presented decreased values, although these differences were only statistically significant in the PU model (Figure 5). In addition, significant correlations were observed between global network features and neurobehavioral results, especially in the OFBT variables (Supplementary Table S3). 


\section{Discussion}

Our results showed that FGR models induced functional impairments in the neonatal and the long-term periods that correlate with structural changes observed by network analysis. Interestingly, these differences were more pronounced in the PU model, suggesting a link between severity of the prenatal insult and the degree of the neurodevelopmental consequences later in life.

Regarding perinatal results, both models induced a reduction of birth weight, but only PU model was related to an increased fetal and early postnatal mortality, reproducing severe forms of human FGR [20]. The same findings were observed in previous animal studies, in which PU was associated with changes in cardiovascular Doppler parameters, leading to increased fetal mortality [9]. This contrasted with the UN models, based either on global nutrient reduction or low-protein diet, which were associated with birth weight reduction with no significant increase in fetal mortality [6, 9, 21].

Neurobehavioral data confirm previous studies showing that both FGR models correlate with neonatal and long-term neurobehavioral impairments, especially for PU cases. During the neonatal period, clinical studies have described neurobehavioral problems related to FRG, including psychomotor delays and cerebral palsy in the most severe cases [22, 23], or subtler neurocognitive difficulties in less severe forms $[24,25]$. Along this line, important motor [26] and olfactory problems [27] were observed in a severe and acute hypoxic-ischemic model in pregnant rabbits, whereas weaker functional disturbances were observed in less severe and chronic PU exposure [28]. At the end of the spectrum, moderate nutrient restriction in pregnant mice has been related to subtle neurobehavioral impairment, such as delayed development of physical and coordinated movements [21, 29]. At the long-term period, reports on infants having suffered from FGR showed neurocognitive difficulties [30] that were even more prevalent in those cases with evident signs of placental insufficiency [31]. In basic research, FGR animal models including UN, a low-protein diet, and PU showed higher degrees of anxiety, reduced social interaction, and depression-related behaviors [16, 29, 32], as well as learning, short-term memory, and attention problems [21, 33-35]. Our results support the notion that severity and type of insult during the prenatal period 
results in a differential effect on neurobehavior, with more remarkable changes in the PU model.

In addition, this study provides new evidence on brain reorganization underlying neurobehavioral and cognition impairments in both models. Global reduction in FA-weighted average strength in both models supported the idea that FGR has an impaired network infrastructure. These results are in line with previous results in a rabbit model, in which average degree of structural brain networks was also decreased [17]. However, altered regional organization evidenced by means of reduced global and local efficiencies was only found in the PU model, demonstrating a more severe effect at this level. Because FA has been related to axonal packing, neuronal density, and myelination of fiber tracts [36], these results suggested that altered network connectivity could be mainly associated with less mature connections. These results are in line with previous studies in humans [37] and animal models [17], showing significantly reduced FA-weighted network efficiencies in FGR at the long-term period.

Overall, this work provides evidence demonstrating that chronic reduction of nutrients with or without a reduction of oxygen, even when started at later stages of pregnancy, still result in a real impact on brain programming. Data presented in this work strengthen the concept that poor nutrition during prenatal life has an impact on later neurobehavioral and cognitive development [38]. Moreover, this study also proves that chronic hypoxia added to undernutrition during the prenatal period has a more severe effect on functional and structural neurodevelopment, thus making the PU suitable to study neurodevelopment consequences of severe forms of FGR. On the contrary, the UN model can be of interest to study effects of less severe forms of FGR.

The main strength of this study is the evaluation of neurodevelopmental consequences in two models of FGR by using the same animal species during the same period. There are a high number of studies describing neurodevelopmental problems in FGR by using different animal species that have provided an undoubted value. However, the rabbit model may have some advantages over the rodent model, as it closely resembles humans in terms of timing of perinatal brain white matter maturation as compared to rats [13]. As in humans, brain maturation begins in the intrauterine period and continues during 
the postnatal period. Apart from that, the suitability of $\mathrm{PU}$ and $\mathrm{UN}$ in rabbits to reproduce human features of FGR has been established [8, 10, 26]. Finally, another strength of this study is the fact that both models followed the same evaluation protocol, in terms of functional test and brain connectivity assessment, offering the possibility to compare the two models.

Limitations of the study include methodological differences between the designs of both models. First, animals from the PU group were delivered at 30 days of pregnancy (near term) by cesarean section, whereas animals from the UN group were allowed to deliver vaginally at 31 days' gestation. In reality, these differences make our results more transferable to clinics, as severe FGR tends to be delivered earlier during pregnancy by means of a cesarean section, whereas less severe cases, which seems to be more accurately reproduced by the UN model, usually are delivered near term by vaginal delivery. Depending on the method of delivery, fetal oxytocin exposure was different. In addition, the difference in the time of birth between the two models has a direct impact on the weight at birth. This difference was evident in the birth weight of the control animals, where controls in the PU model were smaller than the controls in the UN model. Finally, the difference in rearing of the pup could also have an important effect in later neurodevelopment observed in both models. Animals coming from the PU model were fed by a surrogate, whereas animals from the UN model were fed by their mother, who had, however, been undernourished. In order to limit bias due to these design differences, structural and functional differences were assessed, comparing each FGR animal with their matched control, minimizing potential confounders between models. Regarding the brain network extraction, we have applied a tractography method based on DTI appropriate for the 30 gradient directions acquisition. It is known that this technique is less robust in fiber crossing areas than techniques based on highangular resolution diffusion imaging, leading to a lower number of recovered fiber trajectories. However, it has been shown that, from the point of view of case-control studies based on brain network analysis, DTI-based tractography could reduce inter-subject variability, being more sensitive to inter-group variance [39].

\section{Conclusion}


This study demonstrates that sustained intrauterine exposure to placental underperfusion or undernutrition results in functional disturbances and correlates with brain network reorganization. The severity of neurodevelopmental impairment and its association with structural brain reorganization seem to be related to the degree of the prenatal insult, with more remarkable effects in the placental underperfusion model.

The present study adds new evidence regarding neurodevelopmental problems of prenatal origin and improves the understanding of brain programming due to prenatal insults associated with neurobehavioral dysfunctions in FGR. Moreover, it demonstrates the feasibility of using brain network features from diffusion MRI as biomarkers to assess and monitor potential treatments using different experimental models.

\section{Acknowledgments}

We would like to acknowledge Guadalupe Soria and Xavi Lopez Tudela from Experimental 7T MRI Unit of the Institut d'Investigacions Biomèdiques August $\mathrm{Pi}$ i Sunyer (IDIBAPS) for their help in the performance of MRI acquisitions. We are also indebted to Isabel Salas and the rest of the animal housing staff from Hospital Sant Joan de Déu - Animal Housing for their sensitivity in the care of the animal during the procedures. The Image Registration Toolkit was used under Lisence from Ixico Ltd. 


\section{References}

[1] Figueras F, Oros D, Cruz-Martinez R, Padilla N, Hernandez-Andrade E, Botet F, Costas-Moragas C, Gratacos E: Neurobehavior in term, small-forgestational age infants with normal placental function. Pediatrics 2009;124: e934-41.

[2] Sanz-Cortés M, Figueras F, Bonet-Carne E, Padilla N, Tenorio V, Bargalló N, Amat-Roldan I, Gratacós E: Fetal brain MRI texture analysis identifies different microstructural patterns in adequate and small for gestational age fetuses at term. Fetal Diagn Ther 2013; 33:122-129.

[3] Geva R, Eshel R, Leitner Y, Fattal-Valevski A, Harel S: Memory functions of children born with asymmetric intrauterine growth restriction. Brain Res 2006;1117:186-194.

[4] Geva R, Eshel R, Leitner Y, Valevski AF, Harel S: Neuropsychological outcome of children with intrauterine growth restriction: a 9-year prospective study. Pediatrics 2006;118:91-100.

[5] O'Keeffe MJ, O'Callaghan M, Williams GM, Najman JM, Bor W: Learning, cognitive, and attentional problems in adolescents born small for gestational age. Pediatrics 2003;112:301-307.

[6] Vuguin PM: Animal models for small for gestational age and fetal programming of adult disease. Horm Res 2007;68:113-123.

[7] Lang U, Baker RS, Braems G, Zygmunt M, Kunzel W, Clark KE: Uterine blood flow - a determinant of fetal growth. Eur J Obstet Gynecol Reprod Biol 2003; 110(suppl 1):S55- S61.

[8] Eixarch E, Figueras F, Hernández-Andrade E, Crispi F, Nadal A, Torre I, Oliveira S, Gratacós E: An experimental model of fetal growth restriction based on selective ligature of uteroplacental vessels in the pregnant rabbit, Fetal Diagn Ther 2009;26:203-211. 
[9] Eixarch E, Hernandez-Andrade E, Crispi F, Illa M, Torre I, Figueras F, Gratacos E: Impact on fetal mortality and cardiovascular Doppler of selective ligature of uteroplacental vessels compared with undernutrition in a rabbit model of intrauterine growth restriction. Placenta 2011;32:304-309.

[10] López-Tello J, Barbero A, González-Bulnes A, Astiz S, Rodríguez M, Formoso-Rafferty N, Arias-Álvarez M, Rebollar PG: Characterization of early changes in fetoplacental hemodynamics in a diet-induced rabbit model of IUGR. Journal of Developmental Origins of Health and Disease 2015;6(5):454-461.

[11] Rees S, Harding R, Walker D: The biological basis of injury and neuroprotection in the fetal and neonatal brain. Int $\mathrm{J}$ Dev Neurosci 2011; 29:551-563.

[12] Matsuoka T, Mizoguchi $Y$, Serizawa K, Ishikura T, Mizuguchi H, Asano Y: Effects of stage and degree of restricted feeding on pregnancy outcome in rabbits. J Toxicol Sci 2006;31:169-175.

[13] Derrick M, Drobyshevsky A, Ji X, Tan S: A model of cerebral palsy from fetal hypoxia-ischemia. Stroke 2007;38:731-735.

[14] Tan S, Drobyshevsky A, Jilling T, Ji X, Ullman LM, Englof I, Derrick M: Model of cerebral palsy in the perinatal rabbit. J Child Neurol 2005:20:972-979.

[15] Zworykinas SV, Budaevs SV, Zworykin DD: Consistency of skinner box activity in the measure in the domestic rabbit (Oryctolagus cuniculus). Int $\mathrm{J}$ Comp Psychol 1997;159-166.

[16] Illa M, Eixarch E, Batalle D, Arbat-Plana A, Muñoz-Moreno E, Figueras F, Gratacos E: Long-Term Functional Outcomes and Correlation with Regional Brain Connectivity by MRI Diffusion Tractography Metrics in a Near-Term Rabbit Model of Intrauterine Growth Restriction. PLoS One 2013;8:e76453. 
[17] Batalle D, Muñoz-Moreno E, Arbat-Plana A, Illa M, Figueras F, Eixarch E, Gratacos E: Long-term reorganization of structural brain networks in a rabbit model of intrauterine growth restriction. Neuroimage 2014;100:24-38.

[18] Rubinov M, Sporns O: Complex network measures of brain connectivity: uses and interpretations. Neuroimage 2009;52:1059-1069.

[19] de Bruin N, Pouzet B: Beneficial effects of galantamine on performance in the object recognition task in Swiss mice: deficits induced by scopolamine and by prolonging the retention interval. Pharmacol Biochem Behav 2006;85:253260.

[20] M Kady S, Gardosi J: Perinatal mortality and fetal growth restriction. Best Pr Res Clin Obs Gynaecol 2004;18:397-410.

[21] Akitake $\mathrm{Y}$, Katsuragi S, Hosokawa M, Mishima K, Ikeda T, Miyazato M, Hosoda $\mathrm{H}$ : Moderate maternal food restriction in mice impairs physical growth, behavior, and neurodevelopment of offspring. Nutr Res 2015;35:76-87.

[22] Baschat AA: Neurodevelopment after Fetal Growth Restriction. Fetal Diagn Ther 2014;36:136-142.

[23] O'Callaghan ME, MacLennan AH, Gibson CS, McMichael GL, Haan EA, Broadbent JL, Goldwater PN, Dekker GA; Australian Collaborative Cerebral Palsy Research Group: Epidemiologic associations with cerebral palsy. Obstet Gynecol 2011;118:576-582.

[24] Figueras F, Cruz-Martinez R, Sanz-Cortes M, Arranz A, Illa M, Botet F, Costas-Moragas C, Gratacos E: Neurobehavioral outcomes in preterm, growthrestricted infants with and without prenatal advanced signs of brain-sparing. Ultrasound Obstet Gynecol 2011;38:288-294. 
[25] Cruz-Martínez R, Figueras F, Hernandez-Andrade E, Oros D, Gratacos E: Fetal brain Doppler to predict cesarean delivery for nonreassuring fetal status in term small-for-gestational-age fetuses. Obstet Gynecol 2011;117:618-626.

[26] Derrick M, Luo NL, Bregman JC, Jilling T, Ji X, Fisher K, Gladson CL, Beardsley DJ, Murdoch G, Back SA, Tan S: Preterm fetal hypoxia-ischemia causes hypertonia and motor deficits in the neonatal rabbit: a model for human cerebral palsy? J Neurosci 2004;24:24-34.

[27] Drobyshevsky A, Robinson AM, Derrick M, Wyrwicz AM, Ji X, Englof I, Tan S: Sensory deficits and olfactory system injury detected by novel application of MEMRI in newborn rabbit after antenatal hypoxia-ischemia. Neuroimage 2006;32:1106-1112.

[28] Eixarch E, Batalle D, Illa M, Muñoz-Moreno E, Arbat-Plana A, AmatRoldan I, Figueras F, Gratacos E: Neonatal neurobehavior and diffusion MRI changes in brain reorganization due to intrauterine growth restriction in a rabbit model. PLoS One 2012;7:e31497.

[29] Belluscio LM, Berardino BG, Ferroni NM, Ceruti JM, Cánepa ET: Early protein malnutrition negatively impacts physical growth and neurological reflexes and evokes anxiety and depressive-like behaviors. Physiol Behav 2014;129:237-254.

[30] Levine, T.A., Grunau, R.E., McAuliffe, F.M., Pinnamaneni, R., Foran, A., Alderdice, F.A. Early Childhood Neurodevelopment After Intrauterine Growth Restriction: A Systematic Review. Pediatrics. 2015;135:126-141.

[31] Murray E, Fernandes M, Fazel M, Kennedy SH, Villar J, Stein A: Differential effect of intrauterine growth restriction on childhood neurodevelopment: a systematic review. BJOG 2015;122:1062-1072. 
[32] Robinson S, Petelenz K, Li Q, Cohen ML, Dechant A, Tabrizi N, Bucek $\mathrm{M}$, Lust $\mathrm{D}$, Miller $\mathrm{RH}$ : Developmental changes induced by graded prenatal systemic hypoxic-ischemic insults in rats. Neurobiol Dis 2005;18:568-581.

[33] Reyes-Castro LA, Rodriguez JS, Charco R, Bautista CJ, Larrea F, Nathanielsz PW, Zambrano E: Maternal protein restriction in the rat during pregnancy and/or lactation alters cognitive and anxiety behaviors of female offspring. Int J Dev Neurosci 2012;30:39-45.

[34] Valadares CT, Fukuda MT, Françolin-Silva AL, Hernandes AS, Almeida SS: Effects of postnatal protein malnutrition on learning and memory procedures. Nutr Neurosci 2010;13:274-282.

[35] Delcour M, Russier M, Amin M, Baud O, Paban V, Barbe MF, Coq JO: Impact of prenatal ischemia on behavior, cognitive abilities and neuroanatomy in adult rats with white matter damage. Behav Brain Res 2012:232:233-244.

[36] Sen PN, Basser PJ: A model for diffusion in white matter in the brain. Biophys J 2005;89:2927-2938.

[37] Fischi-Gómez E, Vasung L, Meskaldji DE, Lazeyras F, Borradori-Tolsa C, Hagmann P, Barisnikov K, Thiran JP, Hüppi PS: Structural Brain Connectivity in School-Age Preterm Infants Provides Evidence for Impaired Networks Relevant for Higher Order Cognitive Skills and Social Cognition. Cereb Cortex 2015;25:2793-2805.

[38] Dauncey MJ, Bicknell RJ: Nutrition and neurodevelopment: mechanisms of developmental dysfunction and disease in later life. Nutr Res Rev 1999;12: 231-253.

[39] Bastiani M, Shah NJ, Goebel R, Roebroeck A: Human cortical connectome reconstruction from diffusion weighted MRI: The effect of tractography algorithm. Neuroimage 2012;62:1732-1749. 


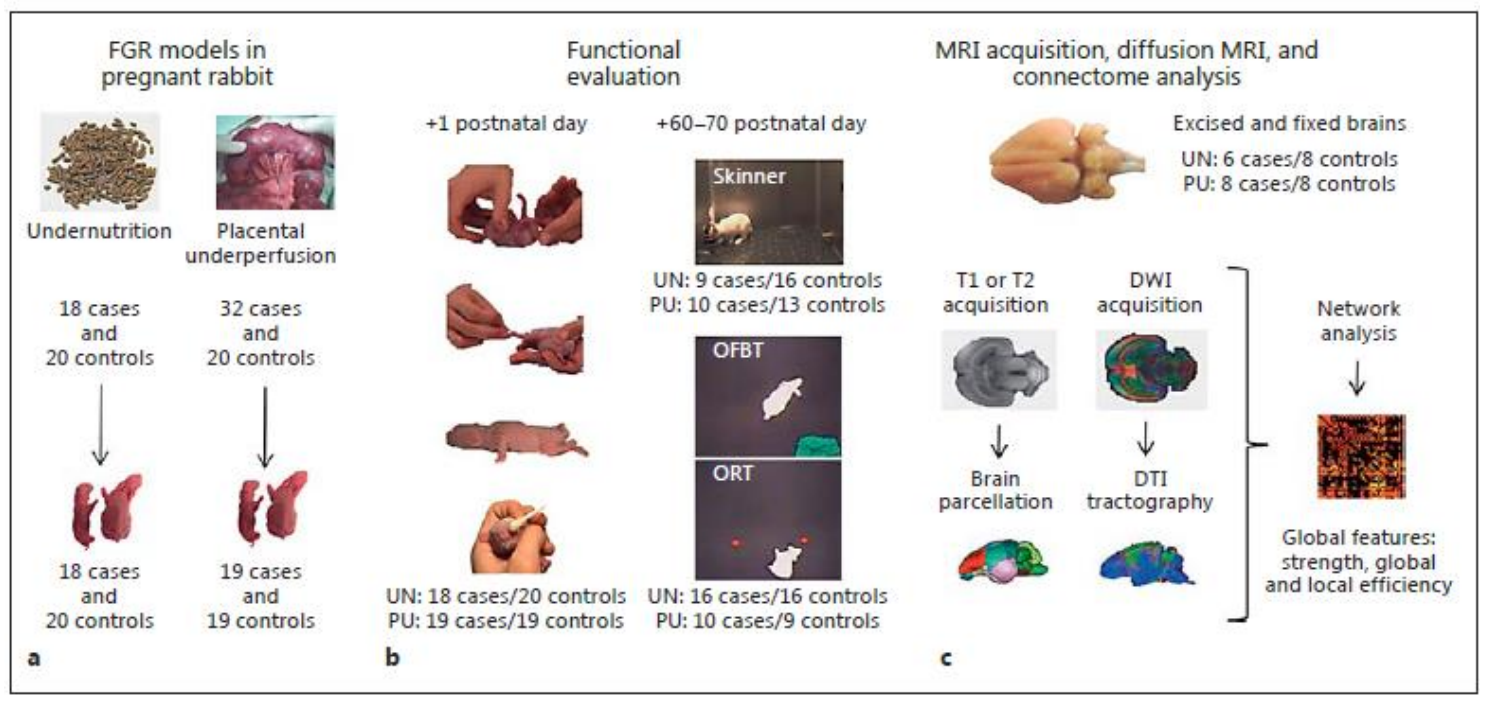

Figure 1: Study design, methods, and flow chart of the animals included

A) Illustrative images and scheme of FGR induction models and number of animals derived from both models.

B) Illustrative images of neurobehavioral tests applied and number of animals evaluated.

C) Illustrative images of MRI acquisition, processing, and connectome analysis.

Abbreviations: $\mathrm{UN}=$ undernutrition; $\mathrm{PU}=$ placental underperfusion; $\mathrm{OFBT}=$ open Field Behavioral test; ORT= object Recognition Task.

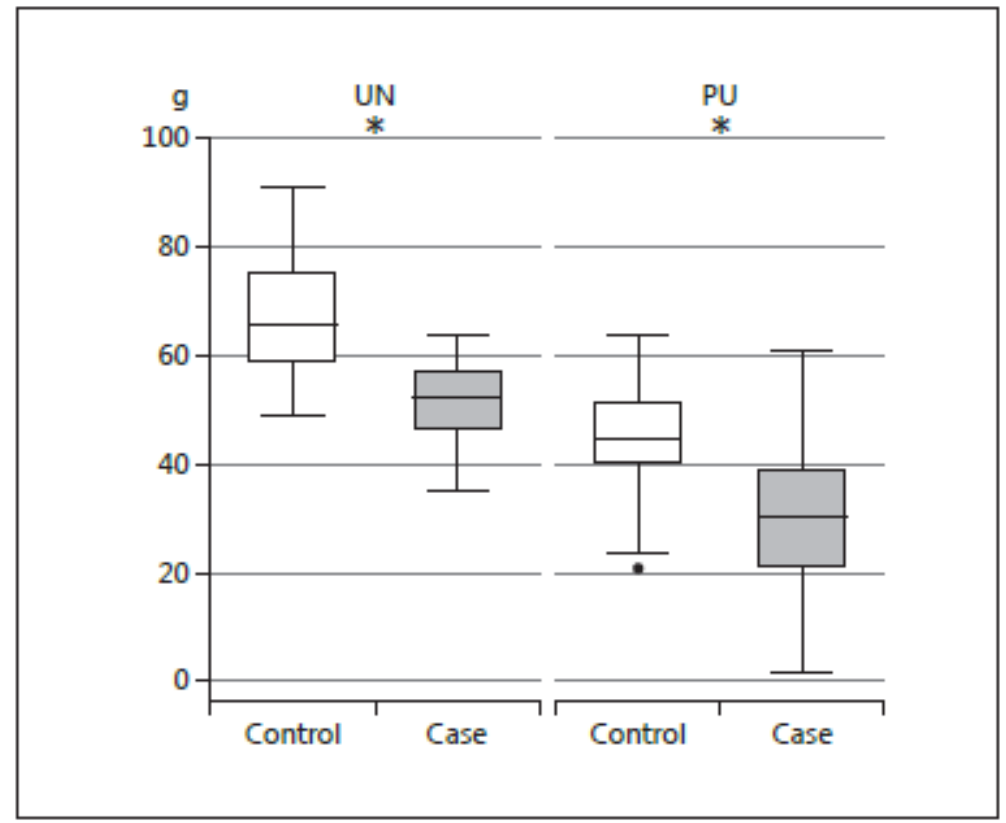

Figure 2: Birth weight differences in study groups

Birth weight (grams) in controls and cases for both models. ${ }^{*} p<0.05$ statistical significance. 


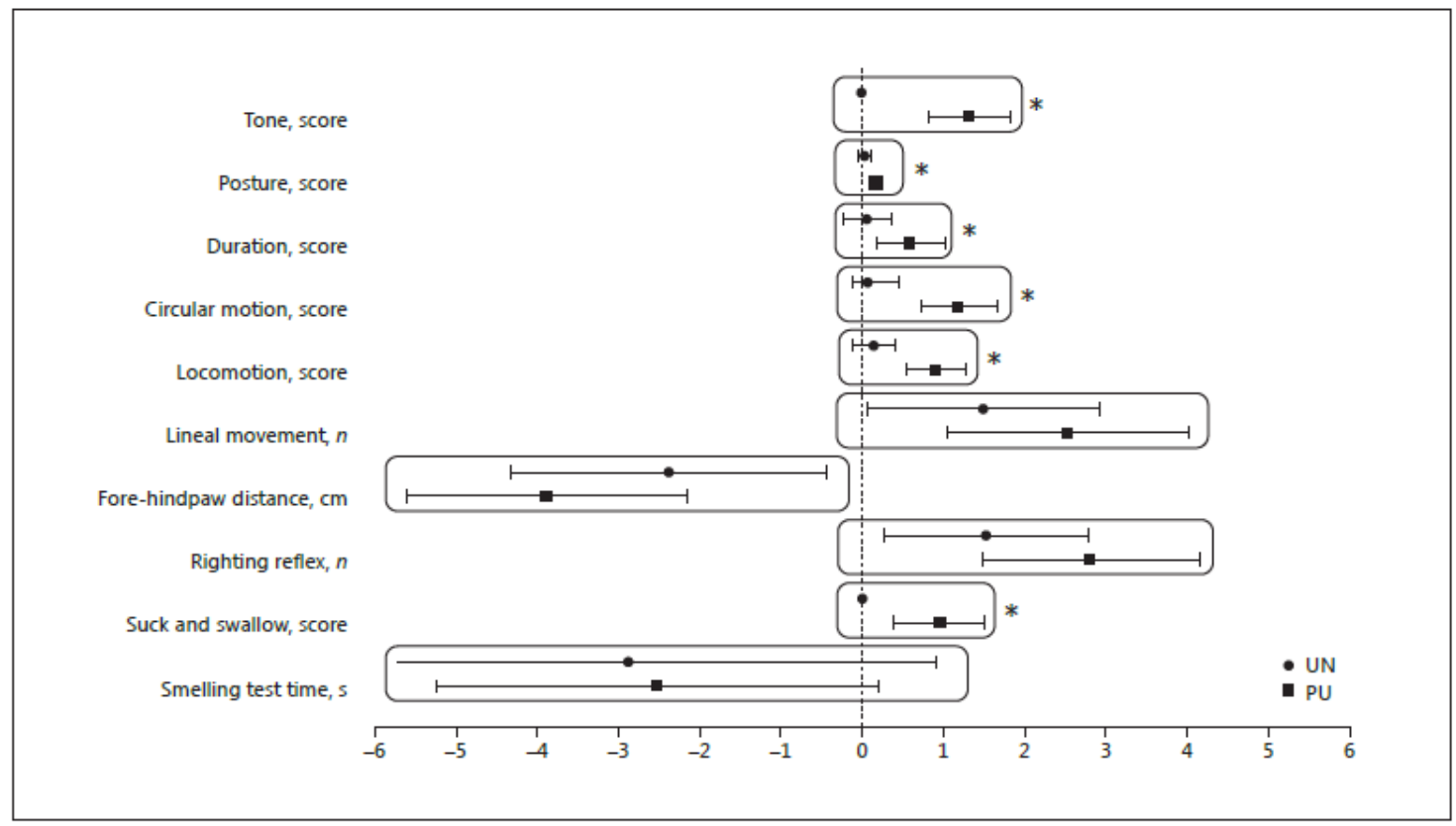

Figure 3: Mean difference of neonatal functional performance in both models

Mean difference (mean result in controls minus mean result in cases) and its 95\% Cl between controls and cases for each model and for each functional logtransformed variable at the neonatal period ${ }^{*} p<0.05$ statistical significance.

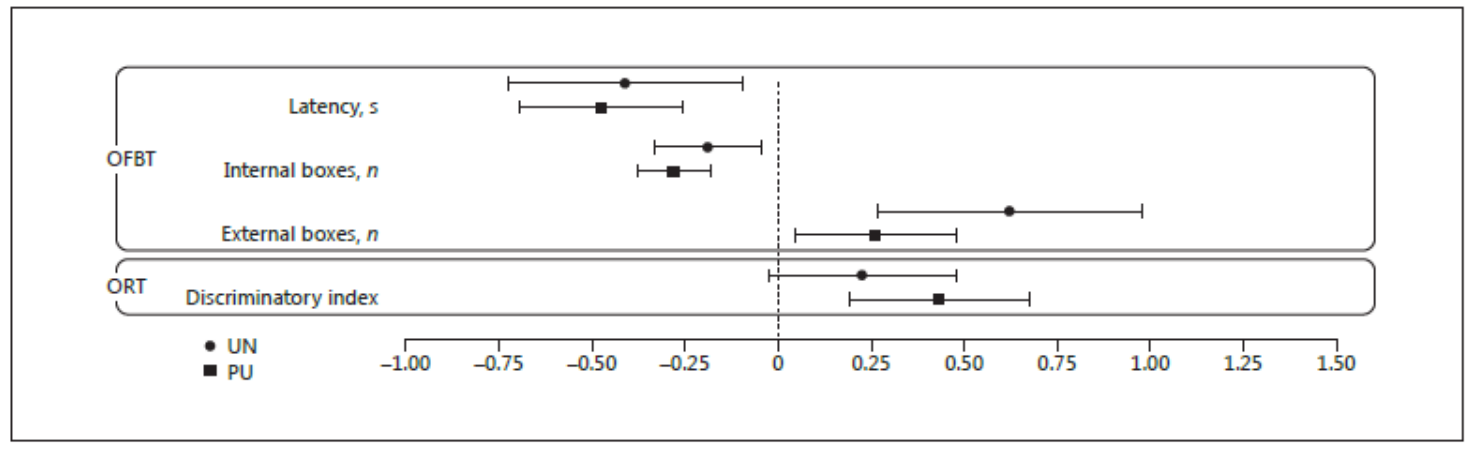

Figure 4: Mean difference of long-term functional performance in both models

Mean difference (mean result in controls minus mean result in cases) and its $95 \% \mathrm{Cl}$ between controls and cases for each model and for each functional logtransformed variable at the long-term period.

Abbreviations: OFBT = open Field Behavioral Test; ORT = object Recognition Task. ${ }^{*} p<0.05$ statistical significance. 


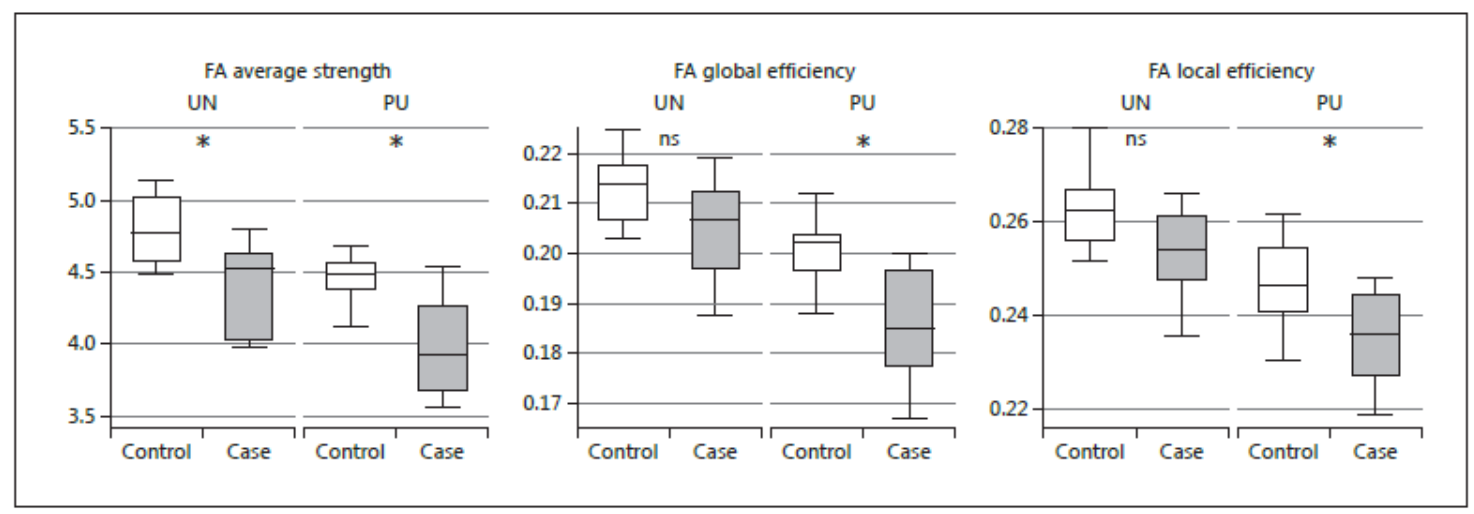

Figure 5: FA-weighted network features

FA-weighted network features in controls and cases for both models, including average strength, and global and local efficiency of weighted FA network. Abbreviations: $U N=$ undernutrition; $P U=$ placental underperfusion. * $p<0.05$ statistical. 